\title{
Pyoderma gangraenosum und Psoriasis pustulosa Barber-Königsbeck bei seropositiver rheumatoider Arthritis
}

\author{
Pyoderma Gangraenosum and Psoriasis Pustulosa Barber-Koenigsbeck in Association with Seropositive \\ Rheumatoid Arthritis
}

Autoren

Institut

\section{Stetter, P. von den Driesch}

Klinik für Dermatologie und Allergologie (Chefarzt: Prof. Dr. med. P. von den Driesch), Zentrum für Hautkrankheiten, Klinikum Stuttgart

\section{Bibliografie}

DOI 10.1055/s-2006-945006

Akt Dermatol 2006; 32;

514-518 @ Georg Thieme

Verlag KG Stuttgart · New York ISSN 0340-2541

Korrespondenzadresse Prof. Dr. med. Peter von den Driesch

Klinik für Dermatologie und Allergologie, Zentrum für Hautkrankheiten · Klinikum Stuttgart Prießnitzweg 24 70374 Stuttgart pdriesch@kbc-intern.de

\section{Zusammenfassung $\nabla$}

Bei chronischen, schmerzhaft ulzerierenden Hautläsionen stellt das Pyoderma gangraenosum eine wichtige Differenzialdiagnose dar. Es handelt sich um eine pathogenetisch nicht restlos geklärte Erkrankung, die vielfach mit internistischen Grunderkrankungen wie beispielsweise chronisch entzündlichen Darmerkrankungen, Paraproteinämien oder rheumatischen Erkrankungen vergesellschaftet ist. Für die Diagnosestellung ist der meist typische klinische Befund, Histologie und direkte Immunfluoreszenz sowie das gute Ansprechen auf eine immunsuppressive

\section{Einleitung}

$\nabla$

Eine wichtige Differenzialdiagnose chronischer Ulcera stellt das sogenannte Pyoderma gangraenosum dar. Dieses wurde erstmals 1930 von Brunsting, Goeckermann und O'Leary als eigene Krankheitsentität beschrieben [1]. Es handelt sich hierbei um eine primär sterile, chronisch ulzerierende Hautaffektion unklarer Ätiologie, die häufig mit einer Grunderkrankung vergesellschaftet ist. Dies können beispielsweise chronisch entzündliche Darmerkrankungen, rheumatische Erkrankungen, oder aber auch monoklonale Gammopathien und andere hämatologische Erkrankungen sowie auch solide Neoplasien sein. Frauen zeigen eine häufigere Erkrankungsinzidenz, das durchschnittliche Erkrankungsalter liegt in der 5. Lebensdekade. Meist kommt es zum Auftreten einer singulären Läsion, die vorzugsweise im Bereich der unteren Extremität lokalisiert ist [2,3]. Die Therapie besteht aus immunsuppressiven Maßnahmen, wie beispielsweise hochdosierten Steroiden, Cyclosporin A, Azathioprin oder Mycophenolatmofetil, aber auch immunmodulierende Ansätze wie intravenöse Immunglobuline, Biologicals bis hin zur
Therapie wegweisend. Im Gegensatz dazu steht die häufig vorkommende Psoriasis mit ihren seltener vorkommenden pustulösen Variationsformen, z.B. der lokalisierten Psoriasis palmoplantaris pustulosa Barber-Königsbeck. Wir stellen eine Patientin vor, bei welcher eine Kombination beider Krankheitsbilder auftrat. Bei beiden Erkrankungen spielen neutrophile Granulozyten eine wichtige pathogenetische Rolle. Bei unserem Patient konnten die Erkrankungen mit einer Kombination von systemischen Steroiden, Mycophenolatmofetil und intravenöser Applikation von Immunglobulinen (IVIG) zur Abheilung gebracht werden.

Plasmapherese kommen je nach möglicher Grunderkrankung in Betracht [4].

Der folgende Fall verdeutlicht die Wichtigkeit der Diagnosestellung, da sich therapeutisch für den Patienten entscheidende Therapiekonsequenzen ergeben und er zeigt, dass es sich nach wie vor beim Pyoderma gangraenosum um ein häufig verkanntes Krankheitsbild handelt. Des Weiteren zeigte die Patientin die unseres Wissens bisher nicht in der Literatur beschriebene pathogenetisch interessante Kombination mit einer lokalisierten pustulösen Form der Psoriasis.

\section{Kasuistik \\ V}

Anamnese

Übernahme einer 81-jährigen, adipösen Patientin in leicht reduziertem AZ bei vorbekannter arterieller Hypertonie, COPD, rheumatoider Arthritis und insulinpflichtigem Diabetes mellitus Typ II. Die Patientin gab an, zunächst einen schmerzhaften roten Knoten im Bereich des rechten Oberschenkels bemerkt zu haben, welcher ohne Trauma spontan entstanden sei. Dieser habe sich innerhalb einer Woche in eine offene Läsion umge- 

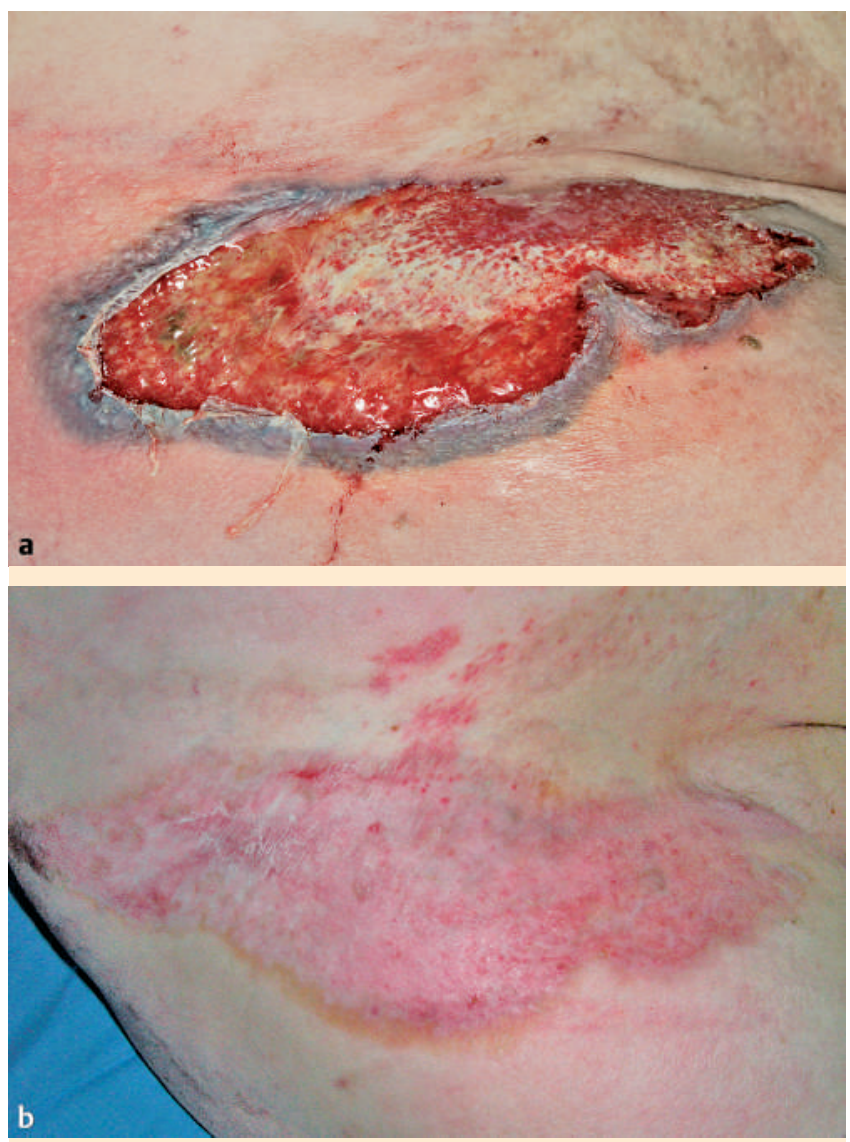

Abb. 1 a Pyoderma gangraenosum rechte Leiste bei rheumatoider Arthritis, $\mathbf{b}$ nach Therapie.

wandelt und habe sich schließlich über einen Zeitraum von 4 Wochen auf eine Größe von ca. $20 \times 10 \mathrm{~cm}$ ausgebreitet. Der äußerst schmerzhafte Verlauf führte zur stationären Notaufnahme in die peripher gelegene chirurgische Klinik, wo die Patientin unter der Diagnose einer fulminanten phlegmonösen Entzündung antibiotisch mit Ciprobay (Ciprofloxacin) und Clont (Metronidazol) intravenös therapiert wurde. Da es hierunter zu einem weiteren schmerzhaften Fortschreiten der Läsion kam, wurde schließlich ab dem 5. Tag zusätzlich die Antibiose um Refobacin (Gentamycin) i.v. erweitert. Ca. 48 Stunden später kam es zu einem generalisierten makulopapulösen Exanthem, weswegen die Patientin schließlich zu uns verlegt wurde. Die Hautveränderungen an den Fußohlen waren der Patientin bis dahin nicht aufgefallen, auch war keine Schuppenflechte anamnestisch bekannt.

\section{Dermatologischer und körperlicher Aufnahmebefund} Im Bereich des rechten Oberschenkels unter Einbeziehung der Beugefalte rechts stellt sich ein scharf begrenztes ca. $33 \times 16 \mathrm{~cm}$ großes Ulcus, teils fibrinös belegt mit starker Wundexsudation und fötidem Geruch dar. Zudem livider, unterminierter Randsaum von ca. 1,5-2 cm Breite übergehend in ein überwärmtes weit in die Peripherie auslaufendes infiltriertes Erythem ( $\odot$ Abb. 1 a).

Im Bereich beider Fußsohlen übergehend zum Malleolus medialis bds. zeigen sich frische Pusteln, teils stecknadelkopfgroß, teils konfluierend, nebst sagokornartig eingetrübten subcornealen Vesikeln und erythematosquamöse Hautveränderungen (๑ Abb. 2a).

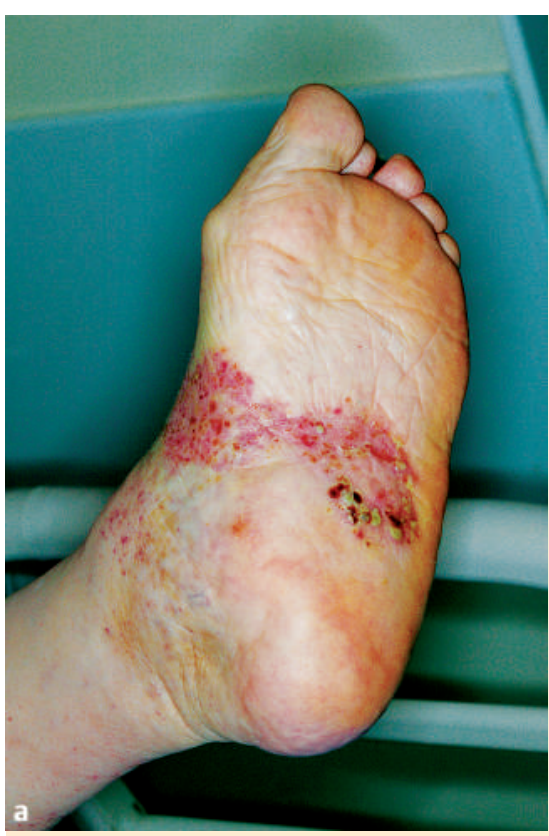

Abb. 2 a Psoriasis pustulosa plantar, b Befund nach Therapie.

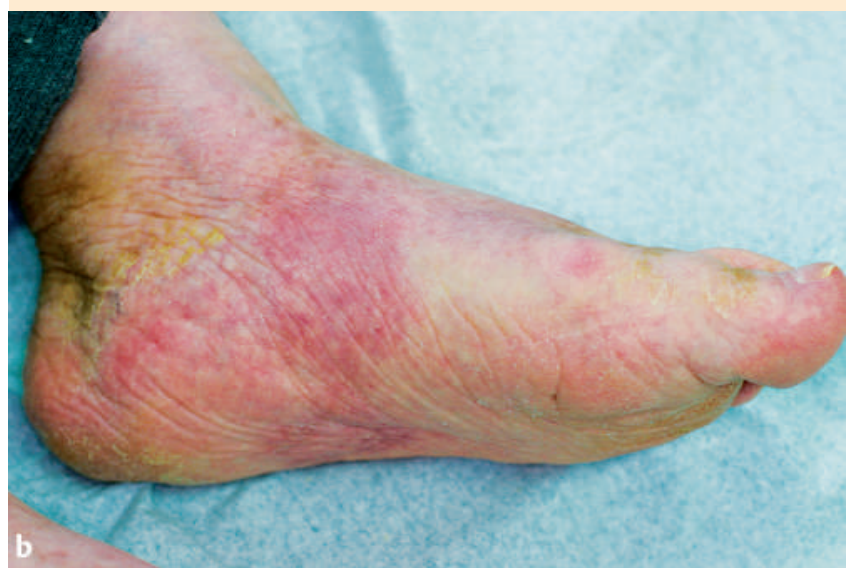

Über das gesamte Integument zeigen sich zudem makulopapulöse Läsionen, teils stecknadelkopfgroß, teils großflächig konfluierend, insbesondere im Bereich des Unterkörpers hämorrhagisch imbibiert.

Nebenbefundlich zeigt sich eine Adipositas per magna bei aktuell leicht reduziertem Allgemeinzustand.

\section{Labor bei Aufnahme:}

Pathologisch verändert:

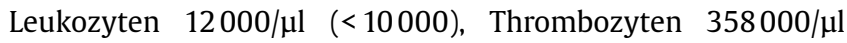
(<350 000), CRP $15 \mathrm{mg} / \mathrm{dl}(<0,5)$, GammaGT $551 \mathrm{U} / \mathrm{l}(<38), \mathrm{LDH}$ 16,9 U/1 (<4,8), Hba1c 7,3\% (<6,3\%), Rheumafaktor IgM $528,8 \mathrm{U} / \mathrm{ml}(<14)$, ANA Endtiter $1: 80$ fein gesprenkelt $(<1: 80)$, BZTP: max. Werte bis $262 \mathrm{mg} / \mathrm{dl}$ nüchtern $(<120)$ unter Steroidmedikation.

\section{Im Normbereich:}

Hb, Natrium, Kalium, Kreatinin, Gesamteiweiß i.S., CK, GOT, GPT, AP, TSH, Quick, PTT, INR, Anti-Streptolysin-Titer, Anti-Staphylolysin-Titer, Anti-Streptodornase, zirkulierende Immunkomplexe, Immunfixationselektrophorese, Kryoglobuline, Anti-DS-DNA, ENA-Profil, c-ANCA, p-ANCA, Yersinien-Serologie, Hepatitis A,B,C-Serologie, Kälteautoantikörper: kein Hinweis für das Vorliegen von irregulären erythrozytären Alloantikörpern oder 
Wärmeautoantikörpern bzw. Kälteautoantikörper, U-Status und Sediment.

Wundabstrich Oberschenkel rechts:

Steril, kein Wachstum von Bakterien oder Pilzen.

Abstrich Pustel plantar rechts:

Steril, kein Wachstum von Bakterien oder Pilzen.

\section{Stuhluntersuchung:}

Kein Wachstum von Parasiten, Salmonellen, Shigellen, Campylobacter oder Yersinien; Hämofec negativ.

Labor bei Entlassung:

Pathologisch verändert:

CRP $2,5 \mathrm{mg} / \mathrm{dl}(<0,5)$, GammaGT $634 \mathrm{U} / \mathrm{l}(<38)$.

\section{Im Normbereich:}

Leukozyten, Thrombozyten, Hb, Natrium, Kalium, Kreatinin, GOT, GPT, AP.

\section{Apparative Untersuchungen}

Röntgen Thorax in 2 Ebenen:

Herz grenzwertig groß, kein Pleuraerguss, keine frischen Infiltrate, kein Hinweis auf Neoplasien, Omarthrose bds.

\section{Abdomensonographie:}

Milz und Niere schemenhaft unauffällig bei vergrößerter Gallenblase, restlicher Oberbauchstatus soweit bei Adipositas beurteilbar o. B.

\section{EKG:}

Sinusrhythmus 79/min., R/S Umschlag in V3, keine spezifischen ERBS.

\section{Therapie und Verlauf}

Die systemische Therapie wurde unverzüglich mit hochdosiertem Steroid, Urbason (Methylprednisolon) $100 \mathrm{mg} / \mathrm{d}$ unter Magenschutz mit Pantozol (Pantoprazol) $40 \mathrm{mg} / \mathrm{d}$ und Osteoporoseprophylaxe (Calcium und Vitamin D3) eingeleitet, die systemische Antibiose wurde beendet. Unter der Steroidmonotherapie kam es zu einer weiteren Verschlechterung des Übernahmebefundes, so dass wir uns 3 Tage später zu einer Erweiterung der Therapie mit Cellcept (Mycophenolatmofetil) in einer Dosis von $1000 \mathrm{mg} 2 \times / \mathrm{d}$ entschieden. Ferner leiteten wir den ersten Zyklus einer intravenösen Immunglobulintherapie mittels Intratect (IVIG), $25 \mathrm{~g}$ Gesamtdosis pro Zyklus ein. Weitere Zyklen folgten in 6 wöchentlichem Abstand. Hierunter kam es zu einem raschen Sistieren der Ausbreitung, so dass eine langsame Steroidreduktion (über Wochen) eingeleitet werden konnte. Die Schmerztherapie erfolgte gemäß WHO Stufenschema, die Lokaltherapie wurde phasengerecht zunächst mit Acticoat, im weiteren Verlauf mit Contreet Ag durchgeführt.

Die Fußsohlen wurden $1 \times / \mathrm{d}$ mit Psorcutanbetasalbe und $1 \times / \mathrm{d}$ mit Chinosol $2 \%$ in Ung. leniens behandelt.

\section{Diskussion \\ $\nabla$}

Das Pyoderma gangraenosum stellt ein seltenes, nach wie vor ätiologisch nicht völlig geklärtes Krankheitsbild dar. Es existiert auch keine einheitliche Leitlinie für dessen Behandlung. Die Diagnosestellung erfolgt meist aufgrund des typischen klinischen Befundes, der Beobachtung von primär sterilen, ohne Therapie zur Chronizität neigenden, eitrigen Ulzerationen, von einem lividen, schmerzhaften, meist unterminierten Randsaum umgeben. In einer Vielzahl der Fälle (ca. 50\%-70\%) lässt sich eine relevante assoziierte Grunderkrankung eruieren, typischerweise aus dem Formenkreis chronisch entzündlicher Darmerkrankungen, rheumatologischer Erkrankungen, Paraproteinämien oder hämoproliferativer Erkrankungen, aber auch solide Neoplasien. Ein weiterer Eckpfeiler in der Diagnosestellung ist die histologische Untersuchung aus dem Randbereich. Hier zeigen sich meist primär neutrophile Infiltrationen der Dermis und Epidermis, teils mit Zeichen einer leukozytoklastischen Vaskulitis. In der direkten Immunfluoreszenz Zeichen einer Immunkomplexvaskulitis mit gefäßgebundenen Ablagerungen von IgA, IgM, IgG, C3 oder C1q. Ein letztes und entscheidendes Kriterium ist das Ansprechen auf immunsuppressive bzw. immunmodulierende Maßnahmen, nicht aber auf eine ausschließlich antiseptische oder antibiotische Therapie. Chirurgische Maßnahmen sind aufgrund des bekannten Pathergiephänomens umstritten bis kontraproduktiv, zumindest ohne begleitende immunsuppressive Therapie [2,5-7].

Die pustulösen Formen der Psoriasis lassen sich in verschiedene Subtypen klassifizieren. Einerseits die generalisierte pustulöse Psoriasis von Zumbusch mit ihrer atypischen Variante, der Erythema anulare centrifugum ähnlichen Psoriasis (EACP), andererseits die lokalisierte Form der Psoriasis palmoplantaris pustulosa Barber-Königsbeck mit ihrer akralen Variante, der Acrodermatitis continua suppurativa Hallopeau [8].

Bei unserer Patientin war die seropositive rheumatoide Arthritis seit vielen Jahren vorbekannt, und mit einer Dauermedikation von 7,5 mg Prednisolon p. o. gut eingestellt. Es ist bekannt, dass die Krankheitsaktivität einer möglichen Grunderkrankung nicht mit der Krankheitsaktivität des Pyoderma gangraenosum korreliert. Dieses kann der jeweiligen Grunderkrankung vorausgehen, oder sich aber nach vielen Jahren erstmals manifestieren $[4,6]$. Interessanterweise kam es bei unserer Patientin gleichzeitig zur Erstmanifestation der Psoriasis pustulosa Barber-Königsbeck und des Pyoderma gangraenosum. In der bisherigen Literatur gibt es vereinzelt Hinweise für das simultane Vorliegen von Pyoderma gangraenosum und Psoriasisarthritis, Psoriasis vulgaris und subcornealer pustulöser Dermatose, nicht aber für Psoriasis pustulosa Barber-Königsbeck und Pyoderma gangraenosum [9-12]. Bei beiden Erkrankungen handelt es sich primär um sterile Infektionen, das heißt um eine vermehrte Ansammlung neutrophiler Granulozyten in der Epidermis bzw. Dermis. Zudem ist bei beiden Erkrankungen die Ätiologie nur teilweise geklärt, der therapeutische Ansatz liegt aber bei immunmodulierenden und immunsupprimierenden Strategien. Beide Erkrankungen zeigen im Bereich der T-Lymphozyten die ausgeprägtesten Veränderungen. Die Psoriasis zeigt zelluläre Infiltrationen in der Epidermis durch CD 8 positive zytotoxische T-Zellen, in der oberen Dermis vorwiegend Infiltrationen durch CD 4 positive T-Zellen. Durch die Sekretion bestimmter Zytokine wie bspw. Interferon $\gamma$, GMCSF, TNF- $\alpha$ und Interleukine 1, 3, 5, 6 und 8 kommt eine direkte Wirkung auf die proliferierenden Keratinozyten zustande [13]. Beim Pyoderma gangraenosum handelt es sich um eine klassi- 
sche neutrophile Dermatose, zu welchen sich unter anderem auch das Sweet-Syndrom und eine Variante, die neutrophile Dermatose des dorsalen Handbereichs (NDDH) zuordnen lässt $[14,15]$. Das Pyoderma gangraenosum zeigt ebenfalls Defekte in der spezifischen humoralen und zellulären Abwehr und dem T-Lymphozyten kommt auch hier eine entscheidende Bedeutung zu, was durch die vermehrte Sekretion von TNF- $\alpha$, Interleukin 1, 2, 8 und Interferon $\gamma$ verdeutlicht wird $[16,17]$. Dadurch lässt sich ableiten, warum beide Erkrankungen auf immunsuppressive und immunmodulierende Maßnahmen ansprechen. Die Therapie der ersten Wahl des Pyoderma gangraenosum besteht aus der hochdosierten Gabe von systemischem Steroid, ggf. in Kombination mit Cyclosporin A. Fälle, die auf diese Kombination nicht ansprechen, oder bei Vorliegen von Kontraindikationen, können alternativ mit Azathioprin, Mycophenolatmofetil, Methotrexat bis hin zu Biologicals (z. B. Infliximab), intravenöser Immunglobulingabe oder Plasmapherese therapeutisch angegangen werden $[4,9,18,19]$. Bei diesen Medikamenten besteht eine weitgehende Überschneidung zu den therapeutischen Optionen, die bei der Psoriasis und der pustulösen Psoriasis zur Anwendung kommen [20-22]. Bei unserem Fall stand das Krankheitsbild des Pyoderma gangraenosum im Vordergrund, so dass sich hier die therapeutische Vorgehensweise ganz darauf bezog. Da eine Monotherapie mit hochdosiertem Steroid $(100 \mathrm{mg} / \mathrm{d})$ allein keinen Erfolg zeigte, musste die Therapie um ein weiteres Immunsuppressivum erweitert werden. Cyclosporin A konnte aufgrund des möglichen Nebenwirkungsprofils bei vorbekanntem insulinpflichtigen Diabetes und arterieller Hypertonie nicht eingesetzt werden. Für Azathioprin wäre bei fulminantem Verlauf die Dauer bis zur vollständigen Entfaltung des Wirkungsprofils zu lange gewesen. Daher entschieden wir uns zur Kombination systemischer Steroide mit Mycophenolatmofetil $(2000 \mathrm{mg} / \mathrm{d})$, bei gleichzeitiger Applikation einer low dose intravenösen Immunglobulingabe ( $25 \mathrm{~g} /$ Zyklus). Beides ist in der Literatur mit Erfolg beschrieben, wobei die Dosierungen der IVIG Therapie durchweg höher $(400 \mathrm{mg} / \mathrm{kg} \mathrm{KG}$, 1. Zyklus; $1 \mathrm{~g} / \mathrm{kg} \mathrm{KG}$ alle weiteren Zyklen, monatlicher Abstand) waren $[18,19,21-23]$. Hierunter kam es zu einem raschen Sistieren der Erkrankung und langsamer Abheilung über die nächsten 6 Monate. Die Patientin erhielt zudem alle 6 Wochen einen IVIG Zyklus mit $25 \mathrm{~g}$ Gesamtdosis über drei Tage verteilt. Unter diesem Regime in Kombination mit 250 mg Mycophenolatmofetil 1-0-1 und Methylprednisolon $8 \mathrm{mg} / \mathrm{d}$ ist die Patientin derzeit (sechs Monate seit Diagnosestellung) in Vollremission und zeigt keine therapeutischen Nebenwirkungen.

Der dargestellte Fall zeigt, dass das Krankheitsbild des Pyoderma gangraenosum ein komplexes Krankheitsbild darstellt, welches nach wie vor oft verkannt wird. Nur durch das generalisierte Arzneimittelexanthem, mutmaßlich auf Gentamycin (eine allergologische Testung ist derzeit unter Immunsuppression noch nicht möglich, aber geplant), gelangte die Patientin in dermatologische Behandlung. Das PG und die Psoriasis pustulosa BarberKönigsbeck zeigen eine enge Beziehung in Bezug auf die möglichen pathogenetischen Zellen "neutrophile Granulozyten“ und „Lymphozyten“, auch wenn beide Erkrankungen bisher nicht simultan erwähnt wurden. Sowohl über die Pathogenese beider Erkrankungen als auch über die vielfältigen Therapieansätze gibt es noch zahlreiche offene Fragen, die es in Zukunft durch systematische Studien und Forschungsarbeiten aufzuklären gilt.

\section{Abstract}

\section{Pyoderma Gangraenosum and Psoriasis Pustulosa} Barber-Koenigsbeck in Association with Seropositive Rheumatoid Arthritis

$\nabla$

The differential diagnosis of chronic and painful ulcerating skin lesions includes Pyderma gangraenosum (PG) which is often associated with systemic disease such as inflammatory bowel disease, paraproteinemia, and rheumatoid arthritis. The diagnosis is usually based on medical history, the clinical, histological and immunohistological findings as well as the fast response to an immunosuppressive therapy. Psoriasis is very common and its pustular variation, the local psoriasis palmoplantaris BarberKoenigsbeck, is a well characterized entity. We present a patient with a combination of PG and Psoriasis palmoplantaris pustulosa. In both diseases neutrophils play a pivotal role in the pathogenesis. In our case, the combination of systemic steroids, mycophenolate mofetil and intravenous immunoglobulin (IVIG) successfully treated both conditions in this patient.

\section{Literatur}

1 Kuner $N$, Hartschuh $W$. Presentation of pyoderma gangraenosum in a dermatologic atlas of the early 19th century. Hautarzt 2000; 51 : $519-523$

2 von den Driesch P. Pyoderma gangraenosum: a report of 44 cases with follow up. Br J Dermatol 1997; 137: 1000-1005

3 Coors EA, von den Driesch P. Pyoderma gangraenosum in a patient with autoimmune haemolytic anaemia and complement deficiency. Br J Dermatol 2000; 143: 154-156

4 Reichrath J, Bens G, Bonowitz A, Tilgen W. Treatment recommendations for pyoderma gangraenosum: an evidence-based review of the literature based on more than 350 patients. J Am Acad Dermatol 2005; 53: $273-278$

5 Hüner A, Hornstein OP, von den Driesch P. Pyoderma gangraenosum; Klinik, assoziierte Erkrankungen und Therapiemöglichkeiten. Dt Ärztebl 1995; 92: B1467-B1471

6 Brooklyn T, Dunnhill G, Probert C. Diagnosis and treatment of pyoderma gangraenosum. BMJ 2006; 333: $181-184$

7 Conrad C, Trueb RM. Pyoderma gangraenosum. JDDG 2005; 3: $334-$ 342

8 Braun-Falco O, Berthold D, Ruzicka T. Psoriasis pustulosa generalisataclassification, clinical aspects and therapy. Review and experiences with 18 patients. Hautarzt 1987; 38: 509-520

9 Spangler JG. Pyoderma gangraenosum in a patient with psoriatic arthritis. J Am Borad Fam Pract 2001; 14: 466-469

10 Phan JC, Hargadon AP, Salpeter SR. Association between pyoderma gangraenosum and psoriasis. Lancet 1996; 348: 547

11 Smith DL, White CR Jr. Pyoderma gangraenosum in association with psoriatic arthritis. Arthritis Rheum 1994; 37: 1258 - 1260

12 Chave TA, Hutchinson PE. Pyoderma gangraenosum, subcorneal pustular dermatosis, IgA paraproteinaemia and IgG antiepithelial antibodies. Br J Dermatol 2001; 145: 852-854

13 Prinz JC. The role of T cells in psoriasis. J Eur Ac Derm Venerol 2003; 17: 257-270

14 Walling HW, Snipes CJ, Gerami P, Piette WW. The relationship between neutrophilic dermatosis of the dorsal hands and sweet syndrome. Arch Dermatol 2006; 142: 57-63

15 Duquia RD, Larangeira de Almeida H, Vettorato G, Souza PRM, Schwartz J. Neutrophilic Dermatosis of the dorsal hands: Acral sweet syndrome? Int J Dermatol 2006; 45: 51 - 52

16 Conrad C, Trueb RM. Pyoderma gangraenosum. J Dtsch Dermatol Ges 2005; $3: 334-342$

17 Tanaka N, Fujioka A, Tajima S, Ishibashi A, Hirose S. Elafin is induced in epidermis in skin disorders with dermal neutrophilic infiltration: interleukin 1 beta and tumor necrosis factor alpha stimulate ist secretion in vitro. Br J Dermatol 2000; 143: $728-732$ 
18 Dobson CM, Parslew RA, Evans S. Superficial granulomatous pyoderma treated with intravenous immunglobulin. J Am Acad Dermatol 2003; 48: $456-460$

19 Lee MR, Cooper AJ. Mycophenolate mofetil in pyoderma gangraenosum. J Dermatol Treat 2004; 15: $303-307$

20 Jolles $S$, Hughes J. Use of IGIV in the treatment of atopic dermatitis, urticaria, scleromyxedema, pyoderma gangraenosum, psoriasis and pretibial myxedema. Int Immunpathol 2006; 6 (4): 579-591

21 Gordon TMH, Lebwohl O, George J, Lebwohl MG. Improvement of pyoderma gangraenosum and psoriasis associated with crohn's disease with anti tumor necrosis factor alpha monoclonal antibody. Arch Dermatol 2001; 137: 930-933

22 Kollmann-Grundmann M, Mooser G, Schraeder P, Zollner T, Kaskel P, Ochsendorf F, Boehncke WH, Kerscher M, Kaufmann R, Peter RU. Treatment of chronic plaque-stage psoriasis and psoriatic arthritis with mycophenolate mofetil. J Am Acad Dermatol 2000; 42: 835 - 837

23 Hagman JH, Carrozzo EC, Romanelli P, Chimenti S. The use of high-dose immunoglobulin in the treatment of pyoderma gangraenosum. J Dermatol Treat 2001; 12: 19-22

\section{Buchbesprechung}

Anti-Aging. Von der Antike zur Moderne

R. M. Trüeb

Darmstadt: Steinkopff, 2006. 180 S., kart., 34,95

ISBN 3-7985-1636-7

Alter, Altern und dessen Aspekte sind in aller Munde und beschäftigen rundum. Insbesondere ist die Alternsforschung und die medizinische Kunst bemüht, das Alter möglichst lebenswert zu gestalten und unsere geistigen und körperlichen Funktionen zu erhalten. Vieles wird beschrieben, geraten und findet Verwendung. So ist es willkommen, einmal den Blick rückwärts zu wenden, zurück in die Jahrtausende der Menschheitsgeschichte, und zu erfahren, dass uns diese Probleme immer begleitet haben. So führt Ralph M. Trüeb, der exzellente Fachmann, uns elegant durch Zeiten und Epochen. Schon in den verschiedenen Kulturen des Altertums werden über 100 Jahre alte Menschen aufgeführt, die teilweise unter diesem leiden, teilweise aber wohlerhalten Ratschläge geben und Geheimnisse ahnen lassen. Aber schon Gilgamesch musste anerkennen, dass die Unsterblichkeit den Menschen versagt bleibt, während Werke und Taten das Fortleben in der Erinnerung der Nachgeborenen ausmachen. Das Wunderkraut als Elexir der Jugend wird ihm von einer Schlange wieder abgenommen und auch in den Indischen Weden ist ein solches nicht zu fassen. Es bleibt der Märchen- und Sagenwelt vorbehalten. Die Philosophen des klassischen Altertums, die arabischen Universalgelehrten und auch die Alchemisten, sie alle meist auch Heiler, kümmerten sich vorwiegend um die philosophische und religiöse Bewältigung von Lebensalter, Vergänglichkeit und Jenseits. Vom christlichen Mittelalter bis kurz vor der Neuzeit sind viele Verhaltensmaßregeln von Unkenntnis und Irrglauben geleitet. Und dennoch, im Bereich der Diätetik und Hygiene sind bemerkenswerte Erkenntnisse zu verzeichnen, die bereits von den Philosophen-Ärzten vorweggenommen wurden und in ihren Grundsätzen bis heute Gültigkeit haben. Zudem wurzelt auch die dekorative Kosmetik im Altertum, von den alten Ägyptern entwickelt, im alten Rom verfeinert und in der Renaissance wieder aufgenommen.
Und die Neuzeit umfasst von der Geriatrie alles bis zum Jugendwahn. Das natürliche Altern wird bizarr gesteigert durch exogene Schädigungen, die sich an Haut und Haaren besonders deutlich zeigen. Dies wird in einem eigenen Kapital dargestellt. Aspekt und Erscheinung werden immer wichtiger und führen auch zu extremen Belastungen. Die Reaktionen sind überdeutlich. So hat die psychodermatologische Gruppe aus Giessen 2001 das so genannte Dorian-Gray-Syndrom beschrieben in Anlehnung an den 1891 erschienen Roman von Oscar Wilde. Statt Dorian's Körper altert dessen Portrait und spiegelt somit den natürlichen Alterungsprozess, getrennt vom Träger. Es ist dies eine Karikatur des modernen Jugendwahns mit der Trias: Übermäßige und pathologische Beschäftigung mit der eigenen Erscheinung, Jugendlichkeitswahn und Verwendung von Lifestyle-Angeboten. Dieses Syndrom drückt verminderte psychische Reife aus und kann vom Narzissmus bis zur Perversion gehen.

Der Gang durch die Zeiten wird eingerahmt durch die psychologische Dimension von Doris Lier zum Anfang und über Attraktivität und Alter von Reinhold Bergler am Schluss.

17 s-w-Abbildungen beleben den Zug durch die Menschheitsgeschichte. Anmerkungen und Literaturhinweise, ein Personen- und ein Sachregister ergänzen den Text, so dass man sich gut zurecht findet.

Den Autoren gebührt großes Lob für die lebhafte, kompetente, attraktive und einladende Darstellung der Materie. Man liest, wird warm und kann sich begeistern. Ein Buch, dem größte Verbreitung zu wünschen ist, nicht nur unter Medizinern und „Älteren“, sondern für Jedermann, auch als nettes Geschenk.

E. G. Jung, Heidelberg 